\title{
Fructose-induced severe hypertriglyceridemia and diabetes mellitus: a cautionary tale
}

\author{
Ana Dugic D1, Michael Kryk¹, Claudia Mellenthin², Christoph Braig', Lorenzo Catanese ${ }^{3}$, \\ Sandy Petermann', Jürgen Kothmann ${ }^{3}$ and Steffen Mühldorfer ${ }^{1}$ \\ 1Department for Gastroenterology, Endocrinology and Metabolic Diseases, Bayreuth University Hospital, Friedrich- \\ Alexander University Erlangen-Nuremberg, Bayreuth, Germany, 2Department of Surgery, HFR Fribourg, Fribourg, \\ Switzerland, and 3Department for Nephrology, Angiology and Rheumatology, Bayreuth University Hospital, \\ Friedrich-Alexander University Erlangen-Nuremberg, Bayreuth, Germany
}

\author{
Correspondence \\ should be addressed \\ to A Dugic \\ Email \\ dugica@gmail.com
}

\begin{abstract}
Summary
Drinking fruit juice is an increasingly popular health trend, as it is widely perceived as a source of vitamins and nutrients. However, high fructose load in fruit beverages can have harmful metabolic effects. When consumed in high amounts, fructose is linked with hypertriglyceridemia, fatty liver and insulin resistance. We present an unusual case of a patient with severe asymptomatic hypertriglyceridemia (triglycerides of $9182 \mathrm{mg} / \mathrm{dL}$ ) and newly diagnosed type 2 diabetes mellitus, who reported a daily intake of $15 \mathrm{~L}$ of fruit juice over several weeks before presentation. The patient was referred to our emergency department with blood glucose of $527 \mathrm{mg} / \mathrm{dL}$ and glycated hemoglobin (HbA1c) of 17.3\%. Interestingly, features of diabetic ketoacidosis or hyperosmolar hyperglycemic state were absent. The patient was overweight with an otherwise unremarkable physical exam. Lipase levels, liver function tests and inflammatory markers were closely monitored and remained unremarkable. The initial therapeutic approach included i.v. volume resuscitation, insulin and heparin. Additionally, plasmapheresis was performed to prevent potentially fatal complications of hypertriglyceridemia. The patient was counseled on balanced nutrition and detrimental effects of fruit beverages. He was discharged home 6 days after admission. At a 2-week follow-up visit, his triglyceride level was $419 \mathrm{mg} / \mathrm{dL}$, total cholesterol was $221 \mathrm{mg} / \mathrm{dL}$ and $\mathrm{HbA} 1 \mathrm{c}$ was $12.7 \%$. The present case highlights the role of fructose overconsumption as a contributory factor for severe hypertriglyceridemia in a patient with newly diagnosed diabetes. We discuss metabolic effects of uncontrolled fructose ingestion, as well as the interplay of primary and secondary factors, in the pathogenesis of hypertriglyceridemia accompanied by diabetes.
\end{abstract}

\section{Learning points:}

- Excessive dietary fructose intake can exacerbate hypertriglyceridemia in patients with underlying type 2 diabetes mellitus (T2DM) and absence of diabetic ketoacidosis or hyperosmolar hyperglycemic state.

- When consumed in large amounts, fructose is considered a highly lipogenic nutrient linked with postprandial hypertriglyceridemia and de novo hepatic lipogenesis (DNL).

- Severe lipemia (triglyceride plasma level $>9000 \mathrm{mg} / \mathrm{dL}$ ) could be asymptomatic and not necessarily complicated by acute pancreatitis, although lipase levels should be closely monitored.

- Plasmapheresis is an effective adjunct treatment option for rapid lowering of high serum lipids, which is paramount to prevent acute complications of severe hypertriglyceridemia. 


\section{Background}

Although hypertriglyceridemia is a common feature of T2DM, severe forms with triglyceride (TG) levels exceeding $2000 \mathrm{mg} / \mathrm{dL}$ are extremely rare in these patients, unless accompanied by diabetic ketoacidosis (DKA). Drinking fruit juice is a widely accepted health trend, as it is perceived as a source of vitamins and nutrients. However, fruit beverages are loaded with fructose, a simple sugar with potentially harmful effects. When consumed in high amounts, fructose can cause hypertriglyceridemia, insulin resistance and visceral fat deposition (1). Because commercial fruit juices are often advertised as having a health benefit, it becomes increasingly difficult for the general population to make healthy food choices. Therefore, it is of utmost significance to raise awareness about a negative impact of fruit beverages, especially in diabetic patients who are at an increased risk of serious metabolic disturbances.

The present case highlights the importance of excessive fructose ingestion as a contributory factor for severe hypertriglyceridemia in a patient with newly diagnosed T2DM.

\section{Case presentation}

A previously healthy 58-year-old Caucasian male was referred to the emergency department due to severe lipemia (TG $9182 \mathrm{mg} / \mathrm{dL}$ and cholesterol $1327 \mathrm{mg} / \mathrm{dL}$ ) and elevated blood glucose of $572 \mathrm{mg} / \mathrm{dL}$. The patient reported intentional weight loss of $\sim 14 \mathrm{~kg}$ over the previous 4 weeks, with a diet comprising excessive amounts of commercial fruit juices of about $15 \mathrm{~L}$ per day and almost no solid foods. After having experienced blurred vision and tingling in his toes, he scheduled an appointment with his family physician who uncovered the abovementioned laboratory abnormalities and referred him to our emergency department.

The patient was alert and oriented on admission, with unremarkable vital signs and BMI of $26.9 \mathrm{~kg} / \mathrm{m}^{2}$ (height $182 \mathrm{~cm}$ and weight $89 \mathrm{~kg}$ ). The skin examination did not reveal any xanthomas or xanthelasmas. The patient's past medical history was completely unremarkable, and his family history was negative for diabetes or dyslipidemia. He was not taking any medications and had no history of tobacco, alcohol or illicit drug abuse.

\section{Investigation}

The initial venous blood gas analysis (VBG) revealed a $\mathrm{pH}$ of 7.35 and glucose above $450 \mathrm{mg} / \mathrm{dL}$, exceeding the upper linear analytical limit of the VBG machine at our emergency room. Urinalysis revealed marked glycosuria and the absence of ketonuria (Table 1). Taken together, clinical and laboratory findings confidently ruled out DKA and hyperosmolar hyperglycemic state (HHS).

Due to the extremely lipemic appearance of the centrifuged blood sample (Fig. 1), further serum chemistry analysis was possible only after the first plasmapheresis session was performed. The relevant laboratory values recorded over the hospital course are presented in Table 2. Lipase levels, inflammatory markers and clinical signs of pancreatitis were closely monitored and remained unremarkable throughout the hospital stay. No signs of arterial hypertension or renal function impairment were observed.

The initial HbA1c was $17.3 \%$ (reference range: $4.8-$ 6.0\%). Random C-peptide was taken at the glucose level of

Table 1 Initial laboratory values.

\begin{tabular}{|c|c|c|}
\hline \multicolumn{2}{|l|}{ Laboratory test } & \multirow[t]{2}{*}{ Reference range } \\
\hline Venous blood gas & & \\
\hline $\mathrm{pH}$ & 7.35 & $7.30-7.43$ \\
\hline $\mathrm{pCO}_{2}(\mathrm{mmHg})$ & 53.7 & $38-58$ \\
\hline Glucose $(\mathrm{mg} / \mathrm{dL})$ & $>450 *$ & $70-115$ \\
\hline Base excess (mmol/L) & 3.9 & -1.9 to 4.5 \\
\hline Bicarbonate (mmol/L) & 25 & $22-30$ \\
\hline Anion gap (mmol/L) & 8.7 & $3.0-11.0$ \\
\hline Sodium (mmol/L) & 130 & $135-143$ \\
\hline Potassium (mmol/L) & 4.3 & $3.6-4.5$ \\
\hline Lactate $(\mathrm{mg} / \mathrm{dL})$ & 24 & $<22$ \\
\hline \multicolumn{3}{|l|}{ Blood chemistry } \\
\hline BUN (mg/dL) & 29 & $18-55$ \\
\hline Creatinine $(\mathrm{mg} / \mathrm{dL})$ & 0,96 & $0.81-1.44$ \\
\hline eGFR (mL/min) & $>60$ & $>60$ \\
\hline Bilirubin total (mg/dL) & 0.6 & $<1.2$ \\
\hline AST (U/L) & 30 & $<50$ \\
\hline $\operatorname{ALT}(\mathrm{U} / \mathrm{L})$ & 42 & $<50$ \\
\hline GGT (U/L) & 51 & $<55$ \\
\hline $\operatorname{ALP}(U / L)$ & 48 & $40-130$ \\
\hline Amylase (U/L) & 25 & $30-110$ \\
\hline Lipase (U/L) & 76 & 73-393 \\
\hline \multicolumn{3}{|l|}{ Urine test strip } \\
\hline Specific gravity & 1.020 & \\
\hline Glucose & +4 & Negative \\
\hline Protein & +1 & Negative \\
\hline Ketones & Negative & Negative \\
\hline Leukocyte esterase & Negative & Negative \\
\hline
\end{tabular}

Venous blood gas and urine were analyzed on admission. The displayed blood chemistry values are those obtained after plasmapheresis, as blood sample was too lipemic to be analyzed on admission.

*Value exceeds the upper linear analytical limit of the VBG machine at our emergency room.

ALT, alanine aminotransferase; ALP, alkaline phosphatase; AST, aspartate aminotransferase; BUN, blood urea nitrogen; eGFR, estimated glomerular filtration rate; GGT, gamma-glutamyl transferase. 


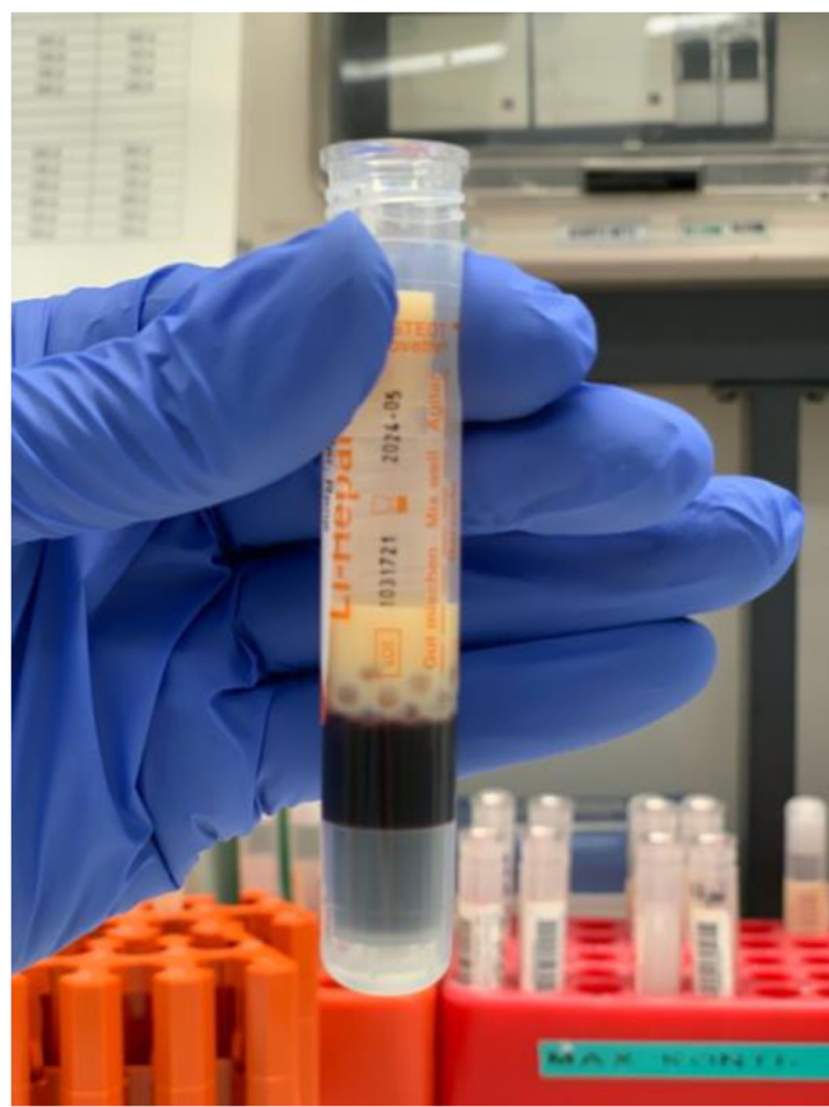

Figure 1

Patient's blood sample after separation with lipemic layer on the top.

$320 \mathrm{mg} / \mathrm{dL}$ after more than $24 \mathrm{~h}$ from the last i.v. insulin application and before plasmapheresis and was sent to the referral laboratory; the C-peptide value was $0.97 \mu \mathrm{g} / \mathrm{L}$ (reference range: 0.81-3.85 $\mu \mathrm{g} / \mathrm{L}$ ). Anti-glutamic acid decarboxylase (anti-GAD), islet cell (anti-ICA) and protein tyrosine phosphatase (anti-IA-2) autoantibodies were also tested before plasmapheresis and were negative, yielding a diagnosis of T2DM.

Lipoprotein electrophoresis was performed 2 days after the second plasmapheresis session (Table 3). The lipid constellation corresponded to type IV dyslipidemia according to the Fredrickson classification of hyperlipoproteinemia.

Abdominal ultrasonography (USG) revealed signs of diffuse hepatic steatosis (USG stage 2). Gall bladder, pancreas, spleen, kidneys and abdominal aorta exhibited no imaging abnormalities. ECG and echocardiography revealed no signs of coronary artery disease.

\section{Treatment}

The patient was admitted to our intensive care unit and treated with i.v. volume and electrolyte resuscitation, insulin, supplemental glucose and prophylactic anticoagulation with heparin. After a steady glucose-level range of $250-300 \mathrm{mg} / \mathrm{dL}$ had been achieved, the patient was transitioned to a s.c. insulin regimen.

To avoid potentially fatal complications of hypercoagulability associated with severe hypertriglyceridemia, the decision was taken to initiate therapeutic plasmapheresis with a $4 \%$ serum albumin solution (polyethylene filter: Plasmaflo OP05 ${ }^{\circ}$ ). Plasmapheresis was performed on the second and third days of admission, and this yielded an immediate reduction of triglyceride level (Fig. 2).

\section{Outcome and follow-up}

During the hospitalization, the patient was counseled on balanced nutrition with emphasis on deleterious effects of uncontrolled fruit juice drinking, such as weight gain, fatty liver and worsening of diabetes and hypertriglyceridemia. In the beginning, the patient was very skeptical toward his diagnosis and was reluctant to initiate antidiabetic therapy, both metformin and insulin. After several counseling sessions, he finally accepted to start insulin but only according to the sliding scale regimen. He was trained on insulin use and self-monitoring of blood glucose by our diabetes team. The patient was discharged home 6 days after admission on s.c. regular insulin according

Table 2 Relevant laboratory values recorded over the hospital course.

\begin{tabular}{|c|c|c|c|c|c|c|c|c|}
\hline & \multicolumn{7}{|c|}{ Hospitalization day } & \multirow[b]{2}{*}{ Reference range } \\
\hline & 1 & 2 & 3 & 4 & 5 & 6 & 2-week follow up & \\
\hline Cholesterol (mg/dL) & 1327 & \# & 334 & 238 & 221 & 247 & 221 & $<200$ \\
\hline Triglyceride (mg/dL) & 9182 & \# & 1603 & 770 & 633 & 698 & 419 & $<200$ \\
\hline HDL-cholesterol (mg/dL) & * & \# & 23 & 17 & 19 & 22 & & $>35$ \\
\hline LDL-cholesterol (mg/dL) & * & * & $\star$ & * & * & * & 133 & $<150$ \\
\hline HbA1c \% & & & 17.3 & & 15.5 & & 12.7 & $4.8-6.0$ \\
\hline HbA1c (IFCC) mmol/mol & & & 166 & & 146 & & 115 & $28.9-42.1$ \\
\hline
\end{tabular}

*Value not computable; \#lipemic.

HbA1c, glycated hemoglobin; HDL, high-density lipoprotein; LDL, low-density lipoprotein. 
Table 3 Lipoprotein electrophoresis performed in the referral laboratory 2 days after the last plasmapheresis sitting.

\begin{tabular}{lllc}
\hline Lipid fraction & & Reference range \\
\hline Triglycerides $(\mathrm{mg} / \mathrm{dL})$ & 851 & $<150$ \\
Cholesterol $(\mathrm{mg} / \mathrm{dL})$ & 267 & & \\
Chylomicrones $(\mathrm{mg} / \mathrm{dL})$ & Negative & & $<30$ \\
VLDL $(\mathrm{mg} / \mathrm{dL})$ & 169 & 17 & $>40$ \\
$\mathrm{HDL}(\mathrm{mg} / \mathrm{dL})$ & 79 & $<115$ \\
$\mathrm{LDL}(\mathrm{mg} / \mathrm{dL})$ & Negative & \\
Lp(a) $(\mathrm{mg} / \mathrm{dL})$ &
\end{tabular}

HDL, high-density lipoproteins, LDL, low-density lipoproteins; Lp(a), lipoprotein (a); VLDL, very low-density lipoproteins.

to standard scale protocol. He was strongly advised to visit a diabetologist for further counseling and therapy optimization. On discharge, $100 \mathrm{mg}$ aspirin daily and 40 mg atorvastatin daily were additionally prescribed. After having obtained lipoprotein electrophoresis results a few days later, we subsequently recommended switching atorvastatin to gemfibrozil $600 \mathrm{mg}$ BID.

At a 2-week follow-up appointment with his family physician, his triglyceride level was $419 \mathrm{mg} / \mathrm{dL}$, total cholesterol was $221 \mathrm{mg} / \mathrm{dL}$ and HbA1c was 12.7\%.

\section{Discussion}

Diabetic dyslipidemia is a common feature of T2DM. It is characterized by a typical combination of elevated serum TG and a decrease in high-density lipoproteins (HDL) (2).

Severe forms (with TG levels exceeding $2000 \mathrm{mg} / \mathrm{dL}$ ) are rarely encountered in patients with T2DM unless they are part of preexisting primary dyslipidemia. When caused by diabetes alone, severe hypertriglyceridemia is almost exclusively accompanied by DKA, which presents as a severe insulin deficit of type 1 diabetes. Less commonly, DKA can also be observed in T2DM, in which case it is either linked to a long-standing disease characterized by insulin depletion or severe insulin resistance.

In this case, the absence of DKA and the presence of non-fasting random serum C-peptide at $0.97 \mu \mathrm{g} / \mathrm{L}(\sim 0.32$ $\mathrm{nmol} / \mathrm{L}$ ) at glucose levels above $300 \mathrm{mg} / \mathrm{dL}$ could exclude hyperinsulinemia or total insulin deficiency as a reason for uncontrolled hypertriglyceridemia (3). The amount of the patient's endogenous insulin has been sufficient to prevent excessive lipolysis and subsequent ketogenesis but not hyperglycemia, which is corroborated by the absence of DKA. Moreover, severe familial hyperlipidemia as an underlying cause of excessive TG levels was almost completely ruled out, as will be discussed in detail later. Even if preexisting hypertriglyceridemia and insulin resistance had been present to some extent, assumed based on the metabolic syndrome features the patient exhibited, this alone could not explain such extreme TG values. It became apparent that an additional mechanism must have been involved in such severe metabolic disturbances.

Surprisingly, severe hypertriglyceridemia in this patient appears to have been induced by excessive intake of fructose contained in commercial fruit juices. Mixed with glucose in approximately equal amounts, fructose is the main constituent of sugar-sweetened beverages. As part of his weight-loss plan (which the authors preferred to attribute to severe polydipsia), the patient ingested $\sim 15$ L of fruit juice per day. Given that an average commercial juice contains 8-11 g sugar per $100 \mathrm{~mL}$, the presumed daily amount of ingested sugar exceeded $1.2 \mathrm{~kg}$; the fructose-to-glucose ratio of commercial juices is $~ 60: 40$, yielding consumption of above $720 \mathrm{~g}$ fructose daily (4). Furthermore, this also led to excessive daily caloric intake, which exceeded daily dietary requirements and could have partly contributed to lipogenesis; however, due to the limited amount of the patient's insulin, this does not seem to have been the primary mechanism of lipid synthesis (5). The patient's low insulin levels may also explain his weight loss, despite his consumption of such a hypercaloric diet.

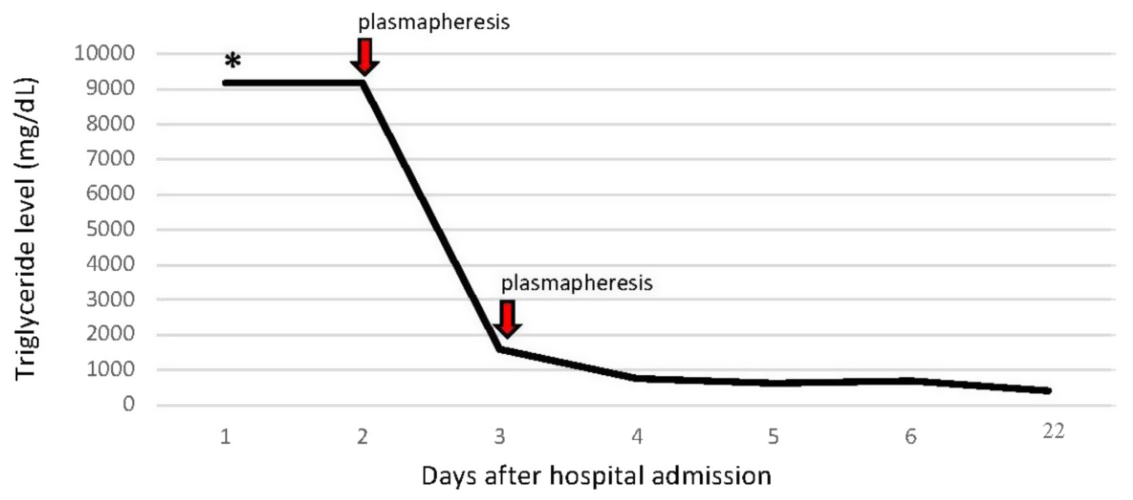

Figure 2

Triglyceride levels during hospital stay. The exact TG level could not be measured in our laboratory before plasmapheresis had been performed, but for graphical presentation, we included the initial value obtained by the referring physician (indicated by asterisk). 
An additional explanation might be weight loss through dehydration caused by uncontrolled diabetes.

Ingesting fructose along with glucose stimulates the flow of fructose to DNL. Unlike glucose, fructose enters glycolysis at a point that bypasses the regulatory control mediated by phosphofructokinase, providing uncontrolled amounts of lipogenic substrates (acetyl-CoA and glycerol3-phosphate) (6); hence, intake of fructose in high amounts leads to TG synthesis from unchecked pathways that are not dependent on insulin. This, in turn, leads to lipid accumulation in the liver, which induces hepatic and peripheral insulin resistance and VLDL production. Moreover, elevated postprandial fructose decreases insulin secretion. As s.c. adipocytes are more sensitive to insulin activation of lipoprotein lipase than the visceral adipocytes, lipid deposition is being diverted from s.c. to visceral fat (7). Recent data suggest a dose-dependent linear relationship between fructose ingestion and an increase in postprandial triglycerides, fasting LDL and 24-h mean acid uric concentration (8), mechanisms that cooperatively promote atherogenesis and contribute to cardiovascular disease (Fig. 3).

The anamnesis, clinical features of metabolic syndrome accompanied by insulin resistance, unremarkable family history and a newly diagnosed T2DM are all highly suggestive of the secondary cause of hypertriglyceridemia in this case. Furthermore, mild preexisting primary hyperlipidemia of Fredrickson type IV could also have been present in this patient (9). Although we could not determine the patient's previous lipid status

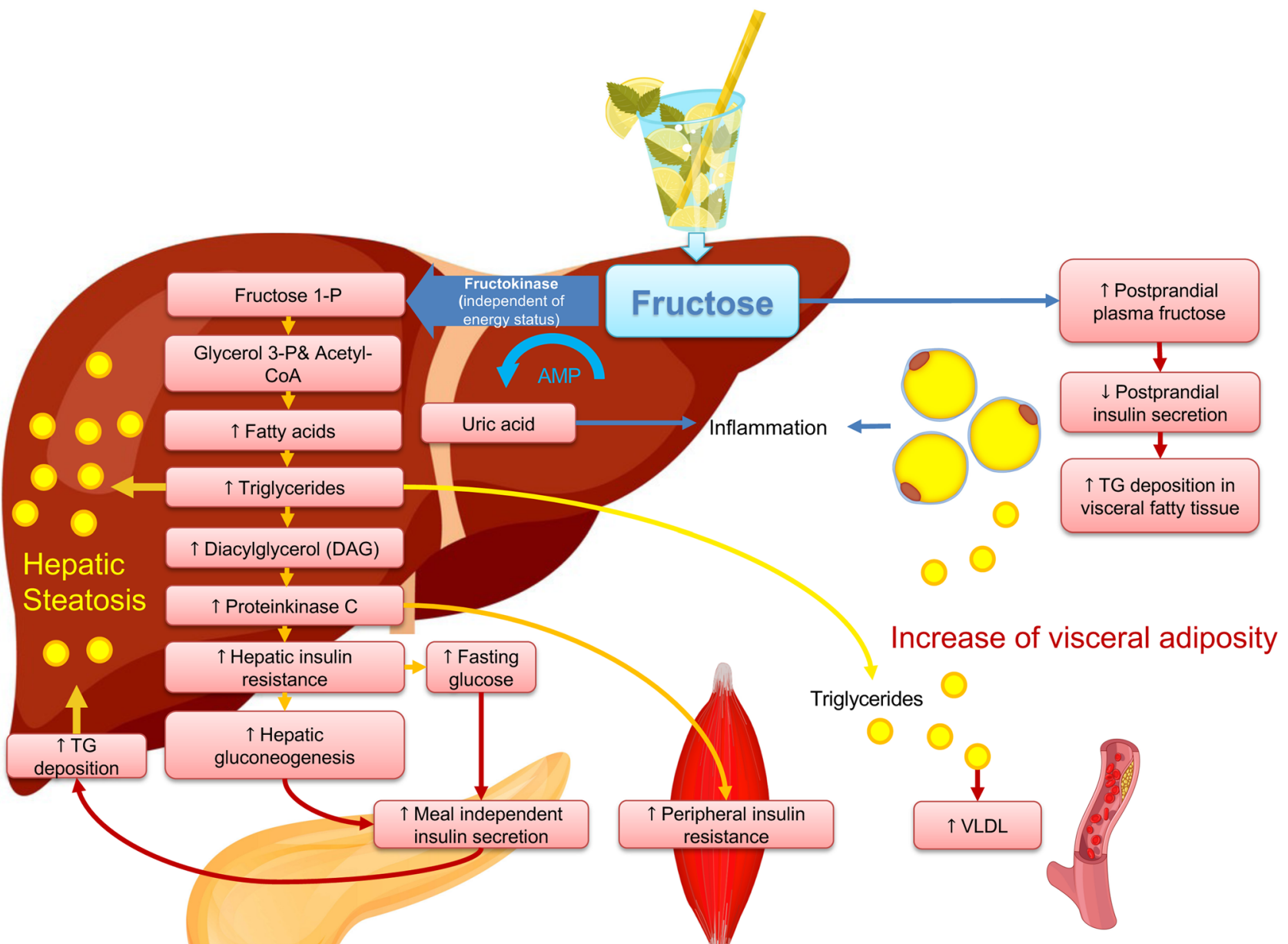

Diabetic metabolic state

\section{Risk of atherosclerosis}

\section{Figure 3}

Metabolic pathways in the state of excessive fructose intake. Consumption of the high-fructose beverages initiates the development of the pathological features associated with metabolic syndrome: dyslipidemia, insulin resistance and increased visceral adiposity. TG, triglyceride; VLDL, very low-density lipoproteins. 
(he considered himself healthy and had not visited a doctor for years), he denied any family history of diabetes, cardiovascular disease or dyslipidemia. No xanthelasmas, tuberous xanthomas or corneal arcus were present on physical examination. Echocardiography and abdominal ultrasonography revealed no signs of atherosclerosis despite his mature age. Finally, lipoprotein electrophoresis and 2-week follow-up values excluded LDL elevation. Consequently, the severe familial forms of hyperlipidemia (such as familial combined hyperlipidemia and familial hypertriglyceridemia) are highly unlikely causes for such extreme TG elevation in this case. Accordingly, we believe that hyperlipidemia was caused by insulin resistance through uncontrolled T2DM and further enhanced by excessive amounts of ingested fructose.

Severe hypertriglyceridemia requires rapid treatment, as it confers a significant risk of acute pancreatitis. It is a rare but well-defined cause of acute pancreatitis (alcohol and biliary etiology being most common), accounting for $2-4 \%$ of all cases (10). Interestingly, our patient did not have pancreatitis, although lipase levels were closely monitored throughout the hospital stay. Accordingly, severe hypertriglyceridemia is not necessarily complicated by acute pancreatitis. On the other side, a prothrombotic state has been reported in association with hypertriglyceridemia (11). To prevent potentially fatal complications of blood hypercoagulability, we employed plasmapheresis. The rapid decline in TG level immediately after plasmapheresis was in accordance with the assumption that hypertriglyceridemia was here partly driven by mechanisms that are not dependent on insulin.

In conclusion, severe hyperlipidemia might have been triggered by excessive fructose intake $(>720$ g per day) in a patient with uncontrolled, newly diagnosed T2DM, an absence of ketoacidosis and measurable serum C-peptide levels. The mechanism involves DNL. Acute pancreatitis does not necessarily complicate severe hypertriglyceridemia. Plasmapheresis is effective in rapid lowering of plasma lipids, which is paramount to prevent complications of hypercoagulability associated with extreme hypertriglyceridemia.

\section{Declaration of interest}

The authors declare that there is no conflict of interest that could be perceived as prejudicing the impartiality of the research reported. The abstract of this manuscript was presented as a virtual poster at the annual European Pancreas Club meeting held online from June 9 to June 12, 2021.

\section{Funding}

This work did not receive any specific grant from any funding agency in the public, commercial or not-for-profit sector.

\section{Patient consent}

Written informed consent for publication of their clinical details was obtained from the patient.

\section{Author contribution statement}

Study conception and design were done by A D, M K and S M. Acquisition of data was done by $A D, M K, L C$, J K. Analysis and interpretation of data were done by A D, M K, C M, C B, J K, S P and S M. Drafting of the manuscript was done by $A D$. Figure illustration was done by $C M$. Critical revision was done by $M K, C M, C B$, J K and S M. Guarantors of the article were A D and S M. All authors read and approved the final manuscript.

\section{References}

1 Geidl-Flueck B, Hochuli M, Nemeth Á, Eberl A, Derron N, Kofeler HC, Tappy L, Berneis K, Spinas GA \& Gerber PA. Fructose- and sucrose- but not glucose-sweetened beverages promote hepatic de novo lipogenesis: a randomized controlled trial. Journal of Hepatology 202175 46-54. (https://doi.org/10.1016/j.jhep.2021.02.027)

2 Haffner SM \& American Diabetes Association. Management of dyslipidemia in adults with diabetes. Diabetes Care 200326 (Supplement 1) S83-S86. (https://doi.org/10.2337/diacare.26.2007.s83)

3 Jones AG \& Hattersley AT. The clinical utility of C-peptide measurement in the care of patients with diabetes. Diabetic Medicine 201330 803-817. (https://doi.org/10.1111/dme.12159)

4 Walker RW, Dumke KA \& Goran MI. Fructose content in popular beverages made with and without high-fructose corn syrup. Nutrition 201430 928-935. (https://doi.org/10.1016/j.nut.2014.04.003)

5 Tappy L \& Rosset R. Health outcomes of a high fructose intake: the importance of physical activity. Journal of Physiology 2019597 3561-3571. (https://doi.org/10.1113/JP278246)

6 Kolderup A \& Svihus B. Fructose metabolism and relation to atherosclerosis, Type 2 diabetes, and obesity. Journal of Nutrition and Metabolism 20152015 823081. (https://doi. org/10.1155/2015/823081)

7 Stanhope KL, Schwarz JM, Keim NL, Griffen SC, Bremer AA, Graham JL, Hatcher B, Cox CL, Dyachenko A, Zhang W, et al. Consuming fructose-sweetened, not glucose-sweetened, beverages increases visceral adiposity and lipids and decreases insulin sensitivity in overweight/obese humans. Journal of Clinical Investigation 2009119 1322-1334. (https://doi.org/10.1172/JCI37385)

8 Stanhope KL, Medici V, Bremer AA, Lee V, Lam HD, Nunez MV, Chen GX, Keim NL \& Havel PJ. A dose-response study of consuming high-fructose corn syrup-sweetened beverages on lipid/lipoprotein risk factors for cardiovascular disease in young adults. American Iournal of Clinical Nutrition 2015101 1144-1154. (https://doi.org/10.3945/ajcn.114.100461)

9 Fredrickson DS \& Lees RS. A system for phenotyping hyperlipoproteinemia. Circulation 196531 321-327. (https://doi. org/10.1161/01.cir.31.3.321)

10 Garg R \& Rustagi T. Management of hypertriglyceridemia induced acute pancreatitis. BioMed Research International 201820184721357. (https://doi.org/10.1155/2018/4721357)

11 Simpson HC, Mann JI, Meade TW, Chakrabarti R, Stirling Y \& Woolf L. Hypertriglyceridaemia and hypercoagulability. Lancet 19831 786-790. (https://doi.org/10.1016/s0140-6736(83)91849-4)

Received in final form 6 October 2021

Accepted 18 October 2021 